\title{
Effect and mechanism of Acoritataninowii Rhizoma and Curcumae Radix on chronic gastritis: a network pharmacology study
}

\section{Ling Zhang}

Piwei Institute

\section{Yunkai Dai}

Piwei Institute

\section{Yuping Li}

Piwei Institute

\section{Weijing Chen}

Piwei Institue

Ruliu Li

Piwei Institute

Ling Hu ( $\nabla$ drhuling@163.com )

Piwei Institute https://orcid.org/0000-0003-3104-8050

\section{Research}

Keywords: chronic gastritis, Acoritataninowii Rhizoma, Curcumae Radix, Network pharmacology

Posted Date: May 21st, 2020

DOI: https://doi.org/10.21203/rs.3.rs-29058/v1

License: (c) (i) This work is licensed under a Creative Commons Attribution 4.0 International License. Read Full License 


\section{Abstract \\ Background}

Chronic gastritis (CG) is an inflammatory disease which is one of the common diseases of the digestive system. To investigate the mechanisms of herbal pair Acoritataninowii Rhizoma(Shichangpu, AR) and Curcumae Radix]Yujin, CR】 in treatment of CG based on the network pharmacology.

\section{Methods}

The possible active ingredients and targets of AR-CR were obtained by the Traditional Chinese Medicine Systems Pharmacology Database and Analysis Platform (TCMSP). The UniProt database was used to query the human gene corresponding to each target protein. The genes related to $C G$ were collected from the GeneCards database, the OMIM database, the DisGeNET database and the PharmGKB database. Intersected the target genes of $A R-C R$ and $C G$, then protein-protein interaction(PPI) network was constructed by STRING website. The overlapped genes were subjected to gene ontology $\square$ GO Ienrichment and Kyoto encyclopedia of genes and genomes $\square$ KEGGロpathway enrichment analyses by David.

\section{Results}

45 intersection genes were obtained, and there were 40 targets in the PPI network for protein interaction, the kernel targets with Degree $\geq 10$ included AKT1, TNF, JUN, MAPK3, MAPK8 and MAPK1. The Go enrichment analysis was mainly related to protein binding, enzyme binding, protein homodimerization activity, etc. The KEGG pathway enrichment analyses mainly involved the Pathways in cancer, TNF signaling pathway, Apoptosis, and VEGF signaling pathway.

\section{Conclusion}

AR-CR might delayed, blocked or reversed the atrophy, intestinal metaplasia, dysplasia and canceration of gastric mucosa by targeting key proteins and signal pathways,achieved the effect of the treatment of CG.

\section{Background}

CG is an inflammatory disease that the epithelium of the gastric mucosa is invaded by various pathogenic factors, and it is one of the common diseases of the digestive system. The recurrence of this disease seriously affects the quality of life of the patient. Chronic atrophic gastritis with intestinal metaplasia and intraepithelial neoplasia increased the risk of gastric cancer, which has attracted more and more attention in clinical ${ }^{[1]}$. Based on endoscopy and pathological examination, it included chronic non-atrophic gastritis and chronic atrophic gastritis ${ }^{[2]}$. Dampness-heat syndrome of spleen-stomach is 
one of the common syndromes of chronic gastritis ${ }^{[1]}$. Dampness-heat disease is extensive, the pathogenesis is complex, the course of disease is long, and the treatment is difficult.

Herbal pair AR-CR is sourced from Wen Bing Quan Shu. The nature and flavor of AR is pungent-warm which could dissipate cold and dispel dampness and strong spleen yang to accelerate transportation and transformation, the qi and flavor of AR is aromatous which could resolve dampness-turbidity. AR is an indispensable herb to treat patients who are dampness-heat of spleen-stomach with yellow-slimy fur, or patients who are cold-dampness encumbering the spleen with white-slimy fur ${ }^{[3]}$. CR acts on qi aspect to move qi and relieve depression, and blood aspect to cool the blood and dissipate stasis. AR combination with CR could move qi and relieve depression, clear the heart and open the orifices,dissipate stasis and resolve phlegm. Herbal pair AR-CR have good clinical effect on patients with spleen-stomach dampnessheat syndrome of CG with the state of anxious or depressed. Professor Lao's Qingzhuo Anzhong Decoction is mainly composed of $A R$ and $C R$, which is quite effective in the treatment of spleen-stomach dampness-heat syndrome of CG ${ }^{[4]}$.

However, the mechanism of herbal pair AR-CR on CG is not clear. In this study, we applied network pharmacology to analyze the effective target of herbal pair AR-CR in the treatment of CG in an integrated and systematic way. Through the topological network analysis, the effective pathways would be deeply explored,and expounded the mechanism of herbal pair AR-CR in the treatment of CG.

\section{Methods}

\section{AR-CR Target prediction}

All ingredients contained in AR and CR were collected from the TCMSP (http://tcmspw.com/tcmsp.php). Screen the retrieved ingredients, and the compound Oral bioavailability $(\mathrm{OB})$ screening thresholds were $\mathrm{OB} \geq 30 \%$, and the compound Drug likeness ( $D L)$ screening thresholds were $D L \geq 0.18$. Candidate compound targets were retrieved by searching the TCMSP. The targets were transformed using the UniProt knowledge database (https://www.uniprot.org/), with the selected species as Homo sapiens. Data were combined to gain gene symbol after removed duplicate items.

\section{Disease target prediction}

We collected gene targets of CG from several sources, included the GeneCards database(https://www.genecards.org/), the OMIM database(https://www.omim.org/), the DisGeNET database(https://www.disgenet.org/) and the PharmGKB database(https://www.pharmgkb.org/).

\section{PPI network of AR-CR}

After the target genes of AR-CR and CG were intersected, the PPI network was constructed using the STRING (https://string-db.org/), which is an update online database, known as revealing target protein interaction analysis, then used Cytoscape3.7.2 for visualization of protein networks. 


\section{Gene enrichment analysis}

The Database for DAVID (https://david.ncifcrf.gov/) server was employed to perform the GO enrichment and the KEGG pathway analysis for the overlapped targets associated with AR-CR and CG. Then, analyzed the influence of overlapped target proteins in gene function and signal pathway, and used Cytoscape3.7.2 to construct the target-pathway network.

\section{Results}

\section{AR-CR Target prediction}

105 compounds of AR and 222 compounds of CR were retrieved by TCMSP. According to the threshold setting, the $\mathrm{OB} \geq 30 \%$ and $\mathrm{DL} \geq 0.18$, and a total of 19 candidate compounds were screened out, including 4 compounds of AR and 15 compounds of CR. Then 130 target proteins of 19 candidate compounds were screened by TCMSP, and the predicted target protein names were transformed into gene names through the Uniprot.

\section{Disease target prediction}

A total of 1057 known therapeutic target symbols of CG were collected from GeneCards database, OMIM database, DisGeNET database and PharmGKB database.

\section{PPI network of AR-CR target}

45 overlapped genes were obtained by intersecting the target genes of AR-CR and CG. The PPI network were constructed using the STRING database, then, the protein network visualization was processed by Cytoscape3.7.2. (Figure 1. PPI network of AR-CR target). There were 40 targets in the PPI network for protein interaction, and there were 5 targets had no protein interactions. In the PPI network, the degree value of each node represents the number of edges connected to each node. The kernel targets with Degree $\geq 10$ included AKT1, TNF, JUN, MAPK3, MAPK8 and MAPK1. 


\section{Gene Ontology (GO) Enrichment Analysis}

The David website was used to perform the GO enrichment analysis of 45 intersection genes to understand molecular function (MF), cellular component (CC) and, biological process (BP). Take the top 10 items of MF, CC, and BP according to $P<0.01$ (Table 1. GO Enrichment Analysis, Figure 2. Bar graph of GO Enrichment Analysis). The Go enrichment analysis showed that herb pair AR-CR mainly involved in molecular function such as protein binding, enzyme binding; cellular component such as cytosol, nucleus, mitochondrion; biological process such as response to drug, response to estradiol.

Table 1 GO Enrichment Analysis 


\begin{tabular}{|c|c|c|c|}
\hline & Term & $P$ & Count \\
\hline \multirow[t]{10}{*}{ MF } & scaffold protein binding & $2.63^{\prime} 10^{-4}$ & 4 \\
\hline & identical protein binding & $1.11^{\prime} 10^{-4}$ & 10 \\
\hline & NADP binding & $1.02^{\prime} 10^{-4}$ & 4 \\
\hline & protein serine/threonine kinase activity & $4.84^{\prime} 10^{-5}$ & 8 \\
\hline & iron ion binding & $4.68^{\prime} 10^{-5}$ & 6 \\
\hline & protein binding & $3.85^{\prime} 10^{-5}$ & 37 \\
\hline & MAP kinase activity & $5.89^{\prime} 10^{-6}$ & 4 \\
\hline & protein homodimerization activity & $2.43^{\prime} 10^{-9}$ & 15 \\
\hline & heme binding & $2.12^{\prime} 10^{-9}$ & 9 \\
\hline & enzyme binding & $2.69^{\prime} 10^{-15}$ & 16 \\
\hline \multirow[t]{10}{*}{$\mathrm{CC}$} & peroxisome & $2.02^{\prime} 10^{-3}$ & 4 \\
\hline & endoplasmic reticulum membrane & $9.44^{\prime} 10^{-4}$ & 9 \\
\hline & nucleus & $4.99^{\prime} 10^{-4}$ & 25 \\
\hline & mitochondrial outer membrane & $4.52^{\prime} 10^{-4}$ & 5 \\
\hline & organelle membrane & $5.69^{\prime} 10^{-5}$ & 5 \\
\hline & membrane raft & $9.57^{\prime} 10^{-6}$ & 7 \\
\hline & extracellular space & $1.89^{\prime} 10^{-6}$ & 15 \\
\hline & mitochondrion & $1.64^{\prime} 10^{-6}$ & 15 \\
\hline & caveola & $4.81^{\prime} 10^{-7}$ & 6 \\
\hline & cytosol & $1.75^{\prime} 10^{-9}$ & 27 \\
\hline \multirow[t]{10}{*}{$\mathrm{BP}$} & aging & $9.62^{\prime} 10^{-9}$ & 9 \\
\hline & regulation of sequence-specific DNA binding transcription factor activity & $4.99^{\prime} 10^{-9}$ & 6 \\
\hline & response to hydrogen peroxide & $3.74^{\prime} 10^{-9}$ & 7 \\
\hline & cellular response to mechanical stimulus & $6.04^{\prime} 10^{-10}$ & 8 \\
\hline & response to hypoxia & $5.24^{\prime} 10^{-10}$ & 10 \\
\hline & response to lipopolysaccharide & $3.43^{\prime} 10^{-10}$ & 10 \\
\hline & response to ethanol & $2.62^{\prime} 10^{-10}$ & 9 \\
\hline & response to drug & $2.58^{\prime} 10^{-10}$ & 12 \\
\hline & lipopolysaccharide-mediated signaling pathway & $1.95^{\prime} 10^{-10}$ & 7 \\
\hline & response to estradiol & $1.63^{\prime} 10^{-12}$ & 10 \\
\hline
\end{tabular}

\section{KEGG Pathway Analysis}

Using the DAVID to performed the KEGG pathway analysis of 45 intersection genes, and 104 enrichment results were obtained. In the pathway enrichment analysis, the top 15 signal pathways are taken according to $P<0.01$ (Table 2. KEGG Pathway Analysis), and a 
bubble chart is drawn using R software (Figure 3. Bubble chart of KEGG Pathway Analysis). The results showed that the herb pair AR-CR could act on VEGF signaling pathway, Apoptosis and so on. The top 15 pathways obtained by KEGG are integrated to draw a target-pathway diagram (Figure 4. target-pathway diagram). Most genes were significantly enriched in the Pathways in cancer, TNF signaling pathway, Apoptosis, and VEGF signaling pathway. The results of KEGG revealed that 4 signaling pathways might be the main signaling pathways related to CG in this network study.

Table 2. KEGG Pathway Analysis

\begin{tabular}{lll}
\hline Term & $P$ & Count \\
\hline Osteoclast differentiation & $6.55^{\prime} 10^{-8}$ & 10 \\
VEGF signaling pathway & $6.41^{\prime} 10^{-8}$ & 8 \\
Influenza A & $6.05^{\prime} 10^{-8}$ & 11 \\
Prostate cancer & $4.30^{\prime} 10^{-8}$ & 9 \\
Non-alcoholic fatty liver disease (NAFLD) & $1.55^{\prime} 10^{-8}$ & 11 \\
Apoptosis & $2.54^{\prime} 10^{-9}$ & 9 \\
Neurotrophin signaling pathway & $1.64^{\prime} 10^{-9}$ & 11 \\
Amyotrophic lateral sclerosis (ALS) & $4.25^{\prime} 10^{-10}$ & 9 \\
Chagas disease (American trypanosomiasis) & $3.96^{\prime} 10^{-10}$ & 11 \\
Tuberculosis & $1.86^{\prime} 10^{-11}$ & 14 \\
Hepatitis B & $1.43^{\prime} 10^{-12}$ & 14 \\
Pathways in cancer & $2.08^{\prime} 10^{-13}$ & 20 \\
Colorectal cancer & $4.34^{\prime} 10^{-14}$ & 12 \\
TNF signaling pathway & $2.60^{\prime} 10^{-14}$ & 14 \\
Toxoplasmosis & $1.09^{\prime} 10^{-15}$ & 15 \\
\hline
\end{tabular}

\section{Discussion}

Chinese medicine has a history of thousands of years, and herb medicines exert extensive biological and pharmacological effects through a variety of compounds and targets. Network pharmacology is an available tool to identify alternative targets for herbal medicines and to develop multi-target drugs. In this study, we analyzed the PPI of AR-CR on the effective targets of CG, and obtained multiple kernel targets including AKT1, TNF, JUN, MAPK3, MAPK8 and MAPK1. In order to clarify how the AR-CR worked through 
these targets, the GO enrichment analysis and the KEGG pathway enrichment analysis were further performed in this study. Through the GO enrichment analysis, we thought that the herb pair AR-CR was able to regulate the protein binding, enzyme binding, response to drug, response to estradiol, etc. The enrichment results of the KEGG signaling pathway showed that the main function of AR-CR might be related to the Pathways in cancer, TNF signaling pathway, Apoptosis, and VEGF signaling pathway, etc.

In the PPI network analysis, one of the key genes such as AKT1 is tightly related to gastrointestinal cancers ${ }^{[5]}$. The activation of AKT1 induces cell proliferation through the stimulation of cell cycle progression and inhibits the intrinsic apoptotic pathway ${ }^{[6]}$. The amount of AKT1 protein can stimulate the proliferation of neoplastic cells,and AKT1 was overexpressed in $8 \%$ of tumors and associated with a trend for a poor overall survival ${ }^{[6]}$. Rescue experiments demonstrated that decreased proliferation and increased apoptosis of gastric cancer cells induced by miRNA4903p overexpression were partially suppressed by AKT1 overexpression [7].

TNF is an important regulator of inflammation and immunity, including TNF - $a$ and TNF - $\beta$. TNF - $a$ is the main inflammatory cytokine, which maintains homeostasis by regulating inflammation, cell proliferation, differentiation, survival and apoptosis ${ }^{[8]}$. The increased expression of TNF-a in the sequential evolution of gastric cancer, the gastric precancerous conditions such as chronic gastritis, intestinal metaplasia, dysplasia and gastric adenocarcinoma patients showed the high expression of TNF-a ${ }^{[9]}$. Xu et al. reported that TNF-a was a major part in inflammatory, infectious and tumor processes, and is pivotal at the early stages of gastric cancer, and TNF-a rs361525 polymorphism is related to the risk of gastric cancer, especially for Asians ${ }^{[10]}$. In the development from CG to gastric cancer, TNF signaling pathway plays an important component in the inflammatory response and tumor ${ }^{[11]}$.

Jun is also known as c-Jun, which played a key component in the tumorigenesis of gastric cancer. Miao et al. reported that KIAA1429 contributed to promoting gastric cancer by regulating c-Jun expression in a m6A independent manner ${ }^{[12]}$. Peng et al. reported that c-Jun could promote the proliferation, metastasis, and invasion of gastric cancer cells in vitro and in vivo by accelerating the expression of FOXK1 at the transcriptional level ${ }^{[13]}$.

MAPK is a group of serine/threonine protein kinases that can be activated by different extracellular stimuli, such as cytokines, neurotransmitters, hormones, cell stress and cell adhesion, which regulate cell growth, differentiation, stress adaptation to the environment, inflammatory response and other important cellular physiological and pathological processes. MAPK3, MAPK1 and MAPK8 are member of the MAPK family. The cell proliferation and invasion of gastric cancer cells were reported to be propelled by the activation of MAPK signaling pathway ${ }^{[14]}$. MAPK3 is also known as ERK1, is an important signal transduction molecule in the ERK/MAPK pathway ${ }^{[15]}$. The ERK/MAPK signal transduction pathway is widely expressed in various tissues and cells, and it can regulate various biological processes such as cell proliferation, cycle, apoptosis, migration, and invasion ${ }^{[16,17]}$. Enhanced functional activity of MAPK3 contributed to the development and progression of gastric cancer ${ }^{[18-20]}$. Knockdown of MAPK1 
expression in gastric cancer cell lines induced cell growth retardation, apoptosis, and suppressed cell migration as well as invasion ${ }^{[21]}$. Diao et al. ${ }^{[22]}$ reported that IncRNA GAPLINC was increased and positively correlated with MAPK1 expression in gastric cancer tumor tissues, additionally, the enhancement of gastric cancer cell proliferation and cell cycle progression by IncRNA GAPLINC was dependent on MAPK1. MAPK1 is a direct target gene of miR-585 that promotes the proliferation and metastasis of gastric cancer ${ }^{[23]}$.MAPK8, which is also known as JNK, is characterized as a pronounced marked protein, is an important cellular pathway triggered in response to DNA damage, and it was also proved to be associated with tumor progression, including cell survival, migration and autophagy ${ }^{[24]}$. Sun et al. ${ }^{[25]}$ reported that MAPK8 could mediate S/G2 phase cycle arrest and apoptosis, and suppress AKT activation in gastric cancer cells, and dysregulated MAPK8 was observed in several cancers, such as glioblastoma, skin cancer, brain tumor and leukemia $[26,27]$.

Increased apoptosis is associated with the development of gastric carcinoma, and $\mathrm{H}$. pylori infection induces apoptosis in gastric epithelial cells by stimulating the host's inflammatory/immune responses [28]. Stimuli and inhibitors for apoptosis may act at targets on the cell membrane via Fas and TNF receptors either in the cytoplasm or in the nucleus ${ }^{[28]}$. The ethanol-induced apoptosis of gastric mucosa could be ascribed to increases in TNF-a level, Bax, and caspase-3 activity ${ }^{[29]}$. A feature of malignancies is an alteration of cell turnover leading to cell hyperproliferation and to deregulation of apoptosis ${ }^{[30]}$. With deepening researches, a sustained inflammatory reaction and abnormal apoptosis of gastric mucosa are confirmed as important causes in the pathogenesis of chronic atrophic gastritis, which have drawn growing attention ${ }^{[31,32]}$. An impaired balance between proliferation and apoptosis was detected in gastric cancer and in atrophic gastritis or intestinal metaplasia in gastric mucosa adjacent to cancer ${ }^{[33]}$.

VEGF contribute to stimulate the growth and differentiation of vascular endothelial cells, is an essential factor in cancer development and progression, its receptors include VEGFR1 and VEGFR2. VEGF could exhibit different levels of expressions in precancerous lesions and gastric cancer, and it is involved in the occurrence and development of gastric cancer ${ }^{[34]}$. Canonical VEGF signaling through VEGFR1/R2 regulates the activities of several kinases and ultimately guides cell proliferation, migration, survival, and vascular permeability during vasculogenesis and angiogenesis ${ }^{[35]}$. Inhibiting the VEGF/VEGFR pathway could cause a rapid and sustained antiangiogenic/antitumor response ${ }^{[36]}$.

\section{Conclusion}

From CG to gastric precancerous lesions, to gastric cancer is a process involving multiple genes, multiple pathways and complicated molecular mechanism. Our study aimed to investigate the mechanisms of AR-CR in the treatment of CG, we performed compounds, targets, pathways prediction and network analysis by using a network pharmacology method. In this study, we thought that herbal pair AR-CR might delayed, blocked or reversed the atrophy, intestinal metaplasia, dysplasia and canceration of gastric mucosa by regulating the above key targets or regulating the above signal pathways, achieved the 
effect of the treatment of CG, which provided important information for the mechanism of AR-CR in the treatment of CG.

\section{Abbreviations}

Chronic gastritis: CG; Acoritataninowii Rhizoma : AR; Curcumae Radix: CR; Traditional Chinese Medicine Systems Pharmacology Database and Analysis Platform :TCMSP; protein-protein interaction network:

PPI; gene ontology: GO; Kyoto encyclopedia of genes and genomes: KEGG; Oral bioavailability: OB; Drug likeness :DL; molecular function :MF; cellular component :CC; biological process :BP;

\section{Declarations}

\section{Acknowledgements}

Not applicable.

\section{Authors' Contributions}

LH conceived this paper. LZ drafted the manuscript. YKD and YPL prepared the data and analyzed the results. WJC and RLL collected information related to this subject. LH revised the manuscript. LZ, YKD, YPL, WJC, RLL, and LH read and approved the final manuscript.

\section{Funding}

This study was funded by National Natural Science Foundation of China (No.81774238هNo. 81373563囚 No.30772689); Innovation team to foster scientific research projects of Guangzhou University of Chinese Medicine, Guangzhou University of Chinese Medicine (No.2014B090902002);Research of double first class discipline of Guangzhou University of Chinese Medicine (2020,No.62,2019,No.5 and 2018,No.6);Construction of first-class discipline of Guangzhou University of Chinese Medicine, Guangzhou University of Chinese Medicine (2017, No.70);Construction of high-level university of Guangzhou University of Chinese Medicine, Guangzhou University of Chinese Medicine (2016,No.64);Innovation team to foster scientific research projects of Guangzhou University of Chinese Medicine, Guangzhou University of Chinese Medicine (No.2016KYTD07).

\section{Availability of data and materials}

The datasets generated and/or analyzed during the current study are available from the corresponding author on reasonable request.

\section{Ethics approval and consent to participate}

Not applicable. 
Not applicable.

\section{Competing interests}

The authors declare no conflict of interest, financial or otherwise.

\section{References}

1. Zhang SS, Tang XD,Huang SP, Bian LQ. Consensus of TCM experts in diagnosis and treatment of chronic gastritis (2017). China Journal of Traditional Chinese Medicine Pharmacy. 2017;32:3060-4. (In Chinese).

2. Fang JY, Du YQ, Liu WZ, Ren JL, et al. Consensus on chronic gastritis in China (2017, Shanghai). Chinese Journal of Gastroenterology. 2017;22:670-87. (In Chinese).

3. Lin CQ, Hu L. Experience of Lao Shaoxian in clinical application of Shichangpu. Journal of Guangzhou University of Traditional Chinese Medicine. 2015;32:147-8 + 151. (In Chinese).

4. OU YH,Lao SX. Influence of Qingzhuo Anzhong Decoction on Gastrin, Motilin and Somatostatin in Patients with Damp-heat Syndrome of Spleen-stomach. Chinese Journal of Integrated Traditional and Western Medicine on Digestion. 2002; 326-328. (In Chinese).

5. Vaseghi Maghvan P, Rezaei-Tavirani M, Zali H, et al. Network analysis of common genes related to esophageal, gastric, and colon cancers. Gastroenterol Hepatol Bed Bench. 2017;10:295-302.

6. Petrini I, Lencioni M, Vasile E, et al. EGFR and AKT1 overexpression are mutually exclusive and associated with a poor survival in resected gastric adenocarcinomas. Cancer Biomark. 2018;21:73141.

7. Yu H, Sun J,JiangS, Xu Y. MicroRNA-490-3p regulates cell proliferation and apoptosis in gastric cancer via direct targeting of AKT1. Exp Ther Med. 2019;17:1330-6.

8. Zhong C, Zhang T, Huang LBX,Xu WH, Chen YN. Mechanism of Banxia Xiexin decoction in the treatment of gastric cancer based on network pharmacology. Chinese Traditional Patent Medicine. 2019;1-12. (In Chinese).

9. Senthilkumar C, Niranjali S, Jayanthi V, Ramesh T,Devaraj H. Molecular and histological evaluation of tumor necrosis factor-alpha expression in Helicobacter pylori-mediated gastric carcinogenesis. $\mathrm{J}$ Cancer Res Clin Oncol. 2011;137:577-83.

10. Xu T, Kong Z,Zhao H. Relationship Between Tumor Necrosis Factor-alpha rs361525 Polymorphism and Gastric Cancer Risk: A Meta-Analysis. Front Physiol. 2018;9:469.

11. Yang LJ, Hu ZP, Li JL, Fan XZ, He W,Pan HF. Identification of genes and pathways in the transformation from chronic gastritis to gastric cancer using bioinformatics analysis. Chinese Archives of Traditional Chinese Medicine. 2019;1-11. (In Chinese).

12. Miao R, Dai CC, Mei L, et al. KIAA1429 regulates cell proliferation by targeting c-Jun messenger RNA directly in gastric cancer. J Cell Physiol. 2020. 
13. Peng $Y$, Zhang $P$, Huang $X$, et al. Direct regulation of FOXK1 by $C$-jun promotes proliferation, invasion and metastasis in gastric cancer cells. Cell Death Dis. 2016;7:e2480.

14. Li C, Liu DR, Li GG, et al. CD97 promotes gastric cancer cell proliferation and invasion through exosome-mediated MAPK signaling pathway. World J Gastroenterol. 2015;21:6215-28.

15. Cao HY, Xiao $\mathrm{CH}$, Lu HJ, et al. MiR-129 reduces CDDP resistance in gastric cancer cells by inhibiting MAPK3. Eur Rev Med Pharmacol Sci. 2019;23:6478-85.

16. Liao T, Wen $D, M a B$, et al. Yes-associated protein 1 promotes papillary thyroid cancer cell proliferation by activating the ERK/MAPK signaling pathway. Oncotarget. 2017;8:11719-28.

17. Buchegger K, Silva R, López J, et al. The ERK/MAPK pathway is overexpressed and activated in gallbladder cancer. Pathology - Research Practice. 2017;213:476-82.

18. Li PY, Lv J, Qi WW, et al. Tspan9 inhibits the proliferation, migration and invasion of human gastric cancer SGC7901 cells via the ERK1/2 pathway. Oncol Rep. 2016;36:448-54.

19. Wang $X, Y u Z$, Zhou Q, et al. Tissue transglutaminase-2 promotes gastric cancer progression via the ERK1/2 pathway. Oncotarget. 2016;7:7066-79.

20. Gao P, Wang S, Jing FZhan J,Wang Y. microRNA-203 suppresses invasion of gastric cancer cells by targeting ERK1/2/Slug/ E-cadherin signaling. Cancer Biomarkers. 2017;19:11-20.

21. Fei $B$, Wu H. MiR-378 inhibits progression of human gastric cancer MGC-803 cells by targeting MAPK1 in vitro. Oncol Res. 2012;20:557-64.

22. Diao L, Wang S, Sun Z. Long noncoding RNA GAPLINC promotes gastric cancer cell proliferation by acting as a molecular sponge of miR-378 to modulate MAPK1 expression. Onco Targets Ther. 2018;11:2797-804.

23. Hu L, Wu H, Wan $\mathrm{X}$, et al. MicroRNA-585 suppresses tumor proliferation and migration in gastric cancer by directly targeting MAPK1. Biochem Biophys Res Commun. 2018;499:52-8.

24. Parra E, Gutierrez L,Ferreira J. Inhibition of basal JNK activity by small interfering RNAs enhances cisplatin sensitivity and decreases DNA repair in T98G glioblastoma cells. Oncol Rep. 2015;33:4138.

25. Sun XL, Zhang XW, Zhai HJ, Zhang D, Ma SY. Magnoflorine inhibits human gastric cancer progression by inducing autophagy, apoptosis and cell cycle arrest by JNK activation regulated by ROS. Biomed Pharmacother. 2020;125:109118.

26. Xu P, Zhang G, Hou S,Sha LG. MAPK8 mediates resistance to temozolomide and apoptosis of glioblastoma cells through MAPK signaling pathway. Biomed Pharmacother. 2018;106:1419-27.

27. Katari SK, Natarajan P, Swargam S, Kanipakam H, Pasala C,Umamaheswari A. Inhibitor design against JNK1 through e-pharmacophore modeling docking and molecular dynamics simulations. Journal of Receptors Signal Transduction. 2016;36:558-71.

28. Xia HH, Talley NJ. Apoptosis in gastric epithelium induced by Helicobacter pylori infection: implications in gastric carcinogenesis. Am J Gastroenterol. 2001;96:16-26. 
29. Raish M, Ahmad A, Ansari MA, et al. Momordica charantia polysaccharides ameliorate oxidative stress, inflammation, and apoptosis in ethanol-induced gastritis in mucosa through NF-kB signaling pathway inhibition. International Journal of Biological Macromolecules. 2018;111:193-9.

30. Zhan QM, Chen J. Cell cycle and tumor translational medicine. Chinese Journal of Clinical Oncology. 2014;41:1-7. (In Chinese).

31. Chen P, Cui Y, Fu QY, Lu YY, Fang JY,Chen XY. Positive relationship between p42.3 gene and inflammation in chronic non-atrophic gastritis. J Dig Dis. 2015;16:568-74.

32. Luo C, Sun Z,Li Z,ZhengL, Zhu X. Notoginsenoside R1 (NGR1) Attenuates Chronic Atrophic Gastritis in Rats. Medical Science Monitor. 2019;25:1177-86.

33. Rosania R, Varbanova M, Wex T, et al. Regulation of apoptosis is impaired in atrophic gastritis associated with gastric cancer. BMC Gastroenterol. 2017;17:84.

34. Zhao WX, Liu ZF, Li XL, Li Z. Correlations of serum homocysteine, VEGF and gastrin 17 with gastric cancer and precancerous lesions. Eur Rev Med Pharmacol Sci. 2019;23:4192-8.

35. Apte RS. Chen DS,Ferrara N. VEGF in Signaling and Disease: Beyond Discovery and Development. Cell. 2019;176:1248-64.

36. Zhang H, Ma RR, Wang XJ, et al. KIF26B, a novel oncogene, promotes proliferation and metastasis by activating the VEGF pathway in gastric cancer. Oncogene. 2017;36:5609-19.

\section{Figures}




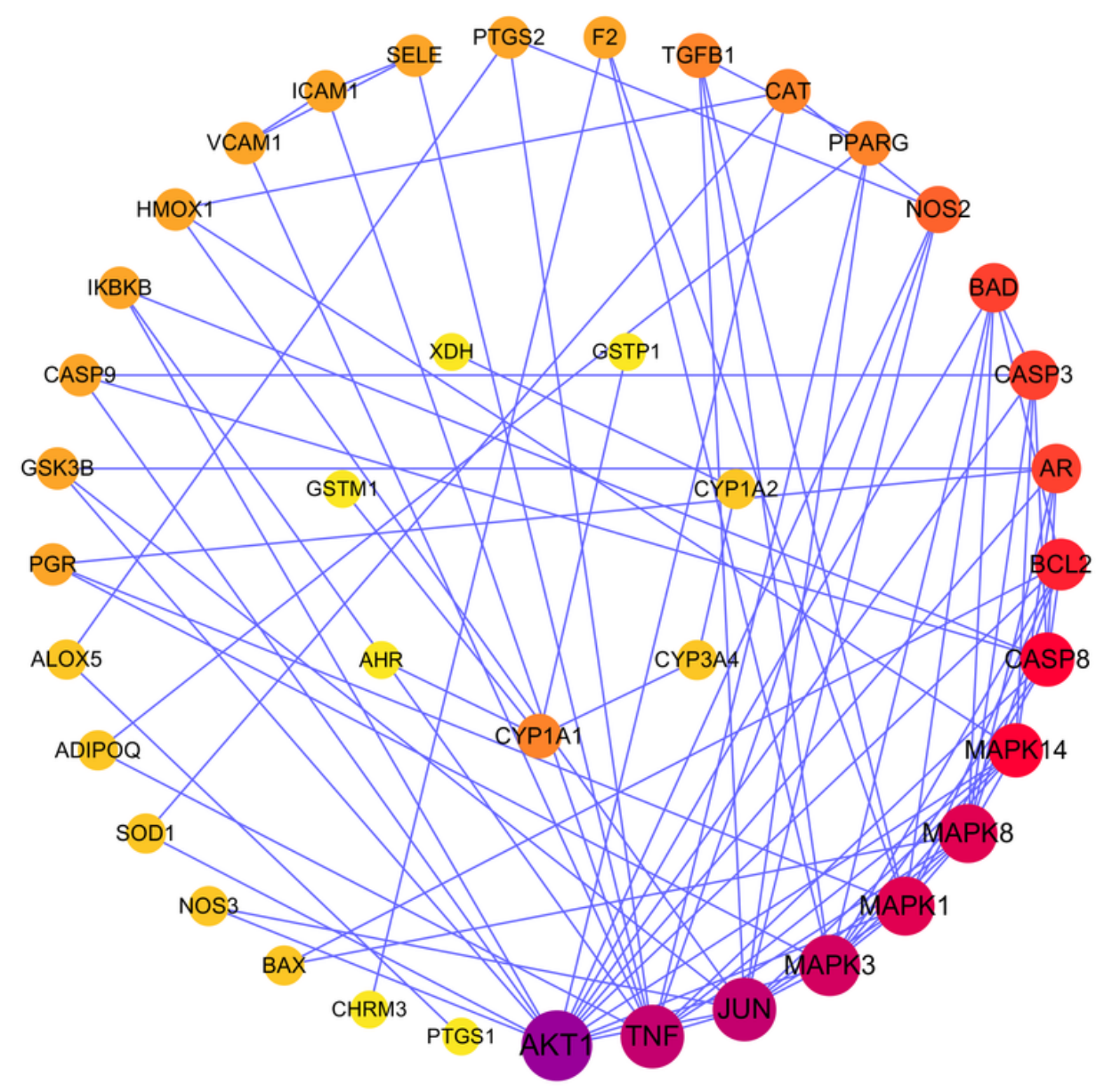

Figure 1

PPI network of AR-CR target 


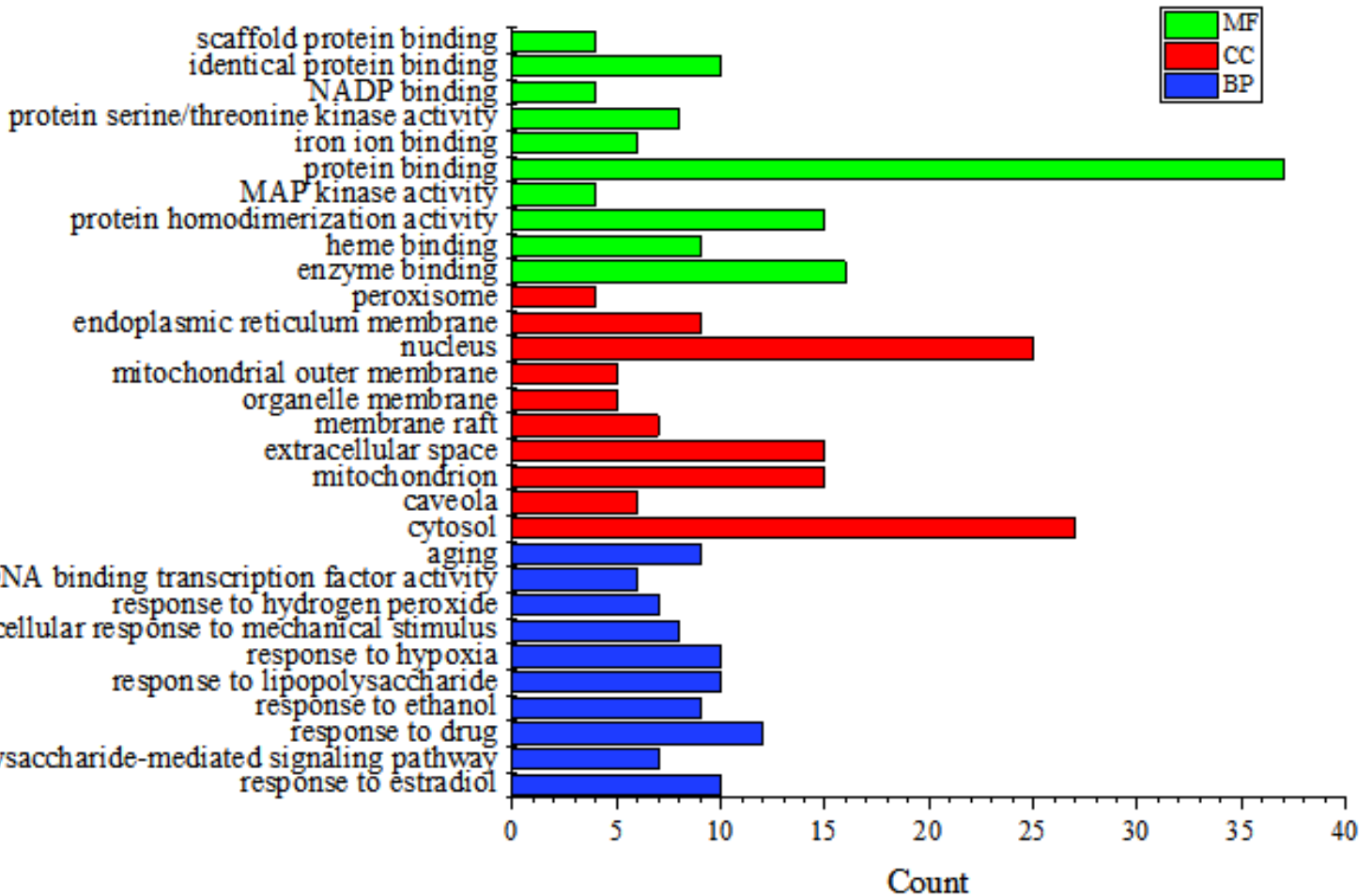

\section{Figure 2}

Bar graph of GO Enrichment Analysis 


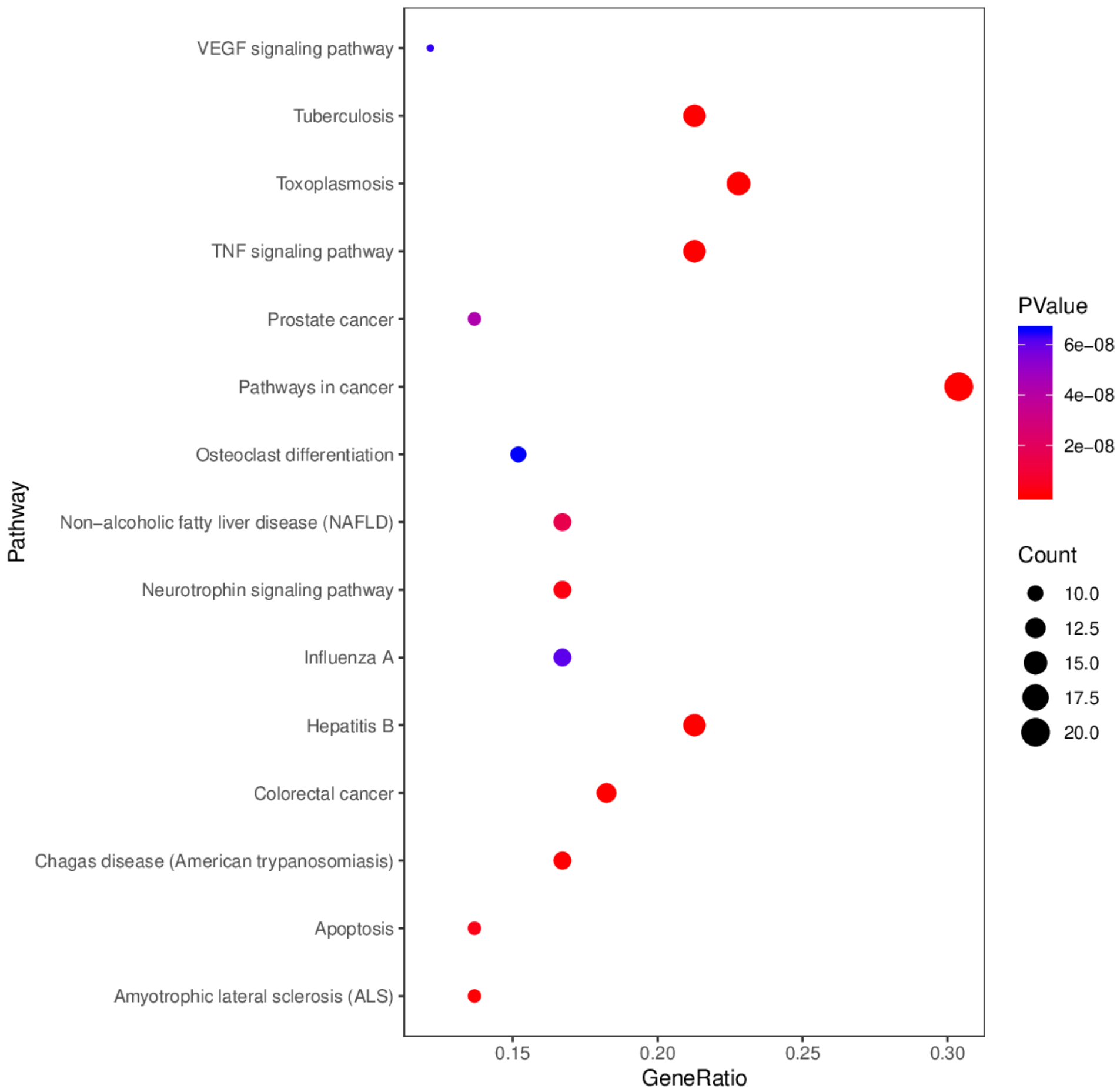

Figure 3

Bubble chart of KEGG Pathway Analysis 


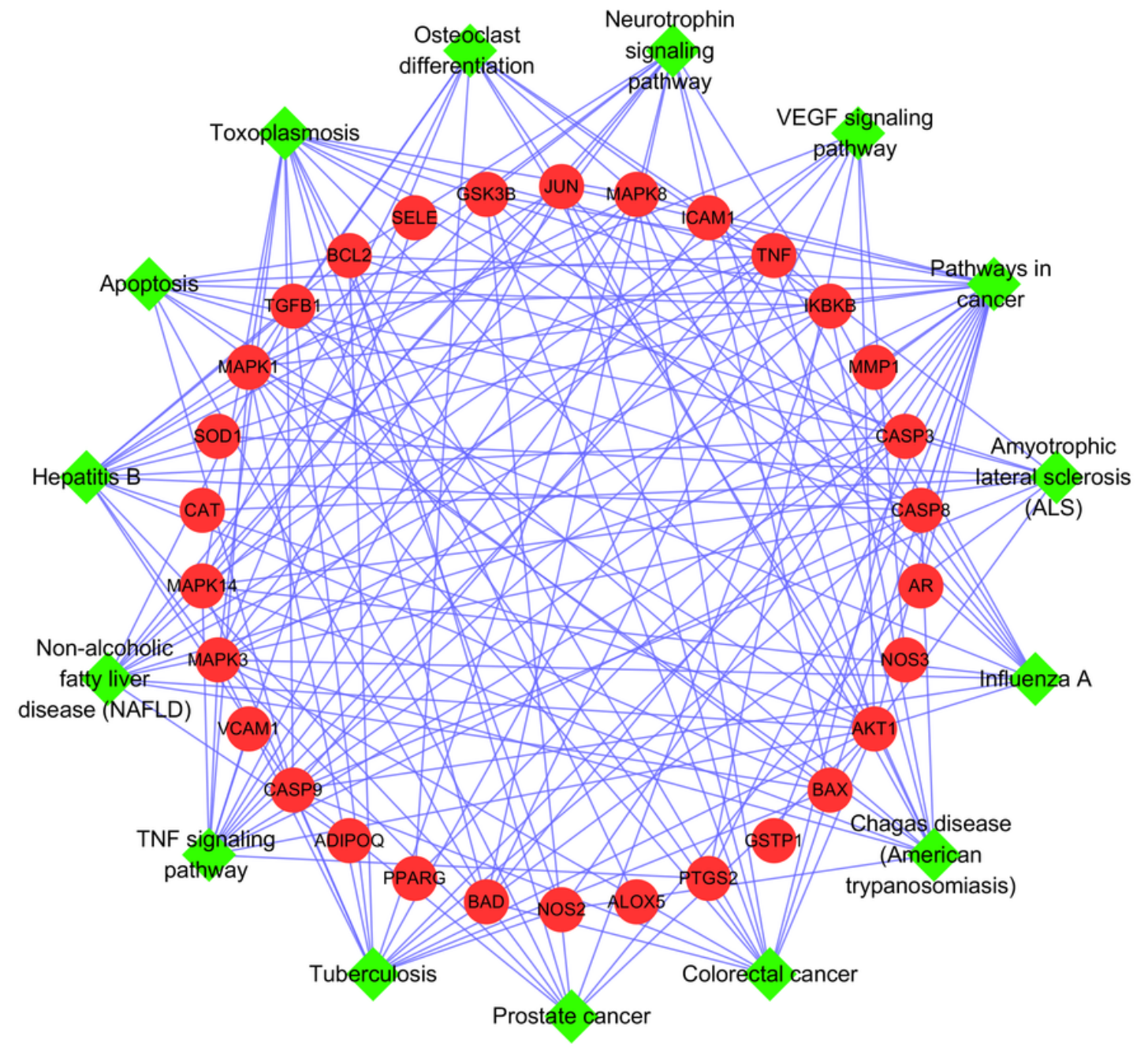

Figure 4

Target-pathway diagram 